\title{
Effects of Renal Impairment on the Pharmacokinetics of Once-Daily Amantadine Extended-Release Tablets
}

\author{
Tina deVries ${ }^{1} \cdot$ Angela Dentiste $^{1} \cdot$ Clifford Di Lea $^{2} \cdot$ Vincent Pichette $^{3} \cdot$ David Jacobs $^{1}$
}

Published online: 24 July 2019

(c) The Author(s) 2019

\begin{abstract}
Background An extended-release formulation of amantadine (Osmolex ER ${ }^{\mathrm{TM}}$, Osmotica Pharmaceutical US LLC) was approved in February 2018 to treat Parkinson's disease and drug-induced extrapyramidal reactions in adults.

Objectives To determine the pharmacokinetic profile of extended-release amantadine in subjects with varying degrees of renal impairment.

Methods Adults with normal renal function (creatinine clearance $>89 \mathrm{~mL} / \mathrm{min} / 1.73 \mathrm{~m}^{2}$ ), moderate renal impairment (creatinine clearance $30-59 \mathrm{~mL} / \mathrm{min} / 1.73 \mathrm{~m}^{2}$ ), or severe renal impairment (estimated glomerular filtration rate $<30 \mathrm{~mL} /$ $\mathrm{min} / 1.73 \mathrm{~m}^{2}$ ) received a single $129-\mathrm{mg}$ dose (160 mg amantadine hydrochloride) of extended-release amantadine. Blood and urine samples for pharmacokinetic analysis were taken at scheduled intervals. A two-compartment pharmacokinetic population model was employed to determine optimum extended-release amantadine dosing in subjects with renal impairment.

Results Following a single oral dose of the 129-mg extended-release amantadine tablet, amantadine plasma concentration increased slowly, reaching a peak at approximately $11 \mathrm{~h}$. Amantadine elimination was reduced in subjects with renal impairment. Renal clearance decreased from 10,965 to $2618 \mathrm{~mL} / \mathrm{h}$ in subjects with severe renal impairment compared to those with normal renal function. Pharmacokinetic modeling and simulation methods were used to recommend the oral administration of 129-mg extended-release amantadine tablets at intervals of 24, 48, 72, 96, 120, or $168 \mathrm{~h}$ depending on the degree of renal function.

Conclusions Renal impairment was associated with reduced amantadine clearance. Based on pharmacokinetic modeling and simulations, dose regimens were recommended for subjects with impaired renal function to provide systemic amantadine exposure similar to subjects with normal renal function taking a once-daily extended-release amantadine tablet.
\end{abstract}

\section{Introduction}

Amantadine is a noncompetitive $N$-methyl-D-aspartate receptor antagonist that is available as an immediate-release (IR) treatment approved for use in idiopathic Parkinson's disease (PD), drug-induced extrapyramidal reactions (also

Electronic supplementary material The online version of this article (https://doi.org/10.1007/s40263-019-00651-1) contains supplementary material, which is available to authorized users.

David Jacobs

djacobs@osmotica.com

1 Osmotica Pharmaceutical US LLC, 400 Crossing Boulevard, Bridgewater, NJ 08807, USA

2 Aclairo Pharmaceutical Development Group, Inc., 1950 Old Gallows Road, Suite 300, Vienna, VA 22182, USA

3 Hôpital Maisonneuve-Rosemont, 5415 Assumption Blvd, Montreal, QC H1T 2M4, Canada

\section{Key Points}

Amantadine absorption is not affected by renal impairment

Increased renal impairment is associated with decreased amantadine clearance

It is recommended that individuals with renal impairment taking $129 \mathrm{mg}$ amantadine ER adhere to the proposed dosing schedule

known as extrapyramidal symptoms), and various strains of influenza [1]. The pharmacodynamics of amantadine IR were studied extensively following its introduction in the 1970s, with more recent work focusing on specialized populations, including PD patients, the elderly, and children with impaired consciousness [2-5]. In February 2018, the US 
Food and Drug Administration approved a new, once-daily, extended-release (ER) formulation of amantadine (OS320) for use in adult subjects to treat PD and drug-induced extrapyramidal reactions. Once-daily oral administration of amantadine ER in the morning delivers an immediate-release amantadine dose followed by slow release of the remaining dose throughout the day [amantadine ER time to maximum plasma concentration $\left(T_{\max }\right)$ is a median of $\left.7.5 \mathrm{~h}\right][6,7]$. Amantadine ER is available in tablets containing 129, 193, or $258 \mathrm{mg}$ amantadine, providing a total dose of 160,240 , or $320 \mathrm{mg}$ amantadine hydrochloride $(\mathrm{HCl})$, respectively.

When administered orally, amantadine is readily absorbed and widely distributed throughout the body, with $67 \%$ of amantadine bound to plasma proteins over a concentration range of $0.1-2.0 \mu \mathrm{g} / \mathrm{mL}$ [8]. It is recommended that subjects with normal renal function begin treatment by taking a oncedaily oral dose of $129 \mathrm{mg}$ amantadine and increase the dose in weekly intervals up to $322 \mathrm{mg}$ maximum [6]. Amantadine elimination is primarily through renal clearance by both glomerular filtration and tubular secretion; thus, in subjects with impaired renal function, amantadine can accumulate in the bloodstream and lead to an increase in adverse events (AEs) and amantadine toxicity $[2,9,10]$.

The primary objective of this phase 1 pharmacokinetic (PK) study was to determine the PK profile following a single 129-mg oral dose of amantadine ER in subjects with moderate and severe renal impairment relative to subjects with normal renal function. In addition, population PK modeling and simulations were performed to identify the recommended amantadine ER tablet dose regimens for subjects with moderate to severe renal impairment.

\section{Methods}

\subsection{Study Design}

This open-label, parallel-group, single-dose, phase 1 PK study was approved by institutional review boards and conducted in compliance with Good Clinical Practice regulations and guidelines as defined by the International Conference of Harmonization. This work complies with ethical principles outlined in the US Code of Federal Regulations, Title 21, Part 50 (21CFR50), and European Union Directive 2001/20/EC. A letter of nonobjection (control number 184363) was obtained from Canadian authorities. All subjects signed informed consent forms after voluntarily agreeing to participate in the study.

Enrolled subjects were divided into two groups of eight based on renal function: group 1, severe renal impairment [estimated glomerular filtration rate (eGFR) $<30 \mathrm{~mL} /$ $\min / 1.73 \mathrm{~m}^{2}$ calculated using the modification of diet in renal disease (MDRD) equation]; group 2, normal renal function [creatinine clearance $(\mathrm{CrCl})>89 \mathrm{~mL} / \mathrm{min}$ calculated using the Cockcroft-Gault equation] [11]. For all subjects, renal function was determined using both MDRD and Cockcroft-Gault equations, but renal insufficiency was identified from only the MDRD calculation, while normal renal function was identified from only the Cockcroft-Gault equation. Despite this utilization of two formulas, no clinically relevant disparities were noted in age or body mass index (BMI) that may have influenced any potential biases. Subjects with renal impairment were not receiving hemodialysis at the time of study participation. Subjects were enrolled in group 2 to match the pooled means for age ( \pm 10 years $)$, gender, and BMI $( \pm 20 \%)$ of those enrolled in group 1. Following a review of PK and safety data from subjects in groups 1 and 2 , subjects with moderate renal impairment (eGFR 30-59 mL/min/1.73 $\mathrm{m}^{2}$; MDRD equation) were also enrolled (group 3; $n=8$ ).

The study design is shown in Fig. S1 of the Electronic supplementary material (ESM). A single dose of $129 \mathrm{mg}$ amantadine ER was administered following a 10-h fast at the clinical site on the morning of day 1 . Subjects refrained from drinking for $1 \mathrm{~h}$ predose to $1 \mathrm{~h}$ postdose and continued fasting for $2 \mathrm{~h}$ postdose. Serial blood samples were collected for $\mathrm{PK}$ analysis through $96 \mathrm{~h}$ postdose for subjects with normal renal function and through $648 \mathrm{~h}$ postdose for subjects with renal impairment. Serial urine samples were collected at intervals up to $96 \mathrm{~h}$ postdose. Including the screening period, the study lasted up to 56 days for renally impaired subjects and up to 34 days for subjects with normal renal function.

\subsection{Study Materials}

Amantadine ER tablets, $129 \mathrm{mg}$ (Osmotica Pharmaceutical US LLC, batch number C1408001).

\subsection{Study Population}

Eligible subjects were males or females (aged 18-80 years) with a BMI of $18-42 \mathrm{~kg} / \mathrm{m}^{2}$ who had not smoked in the preceding 6 months and had a pulse rate of $40-110$ beats $/ \mathrm{min}$ (bpm; groups 1 and 3) or 50-100 bpm (group 2). Subjects were required to test negative for hepatitis $\mathrm{B}$, hepatitis $\mathrm{C}$, and human immunodeficiency virus. Admission of renally impaired subjects with hepatitis $\mathrm{C}$ was allowed at the investigators' discretion if liver enzyme parameters were not considered clinically significant by the investigator.

Subjects were excluded if they had a clinically significant history of hallucinations, depression, or suicidal ideation; displayed clinically significant disease or had abnormalities in medical history, physical examination, serum chemistry, hematology, or urinalysis that might compromise subject 
safety or data quality (with renal impairment groups given consideration for the degree of impairment and presence of comorbidities); were pregnant or nursing; showed evidence of an active infection; had a history of heavy alcohol use (> 3 units per day); were enrolled in another clinical trial; were recent recipients of live attenuated influenza virus vaccine ( 2 weeks prior to day -1 or within $48 \mathrm{~h}$ of dosing); donated blood (56 weeks prior to study day -1 ) or platelets (14 days prior to study day -1$)$; performed rigorous exercise from $48 \mathrm{~h}$ prior to day -1 to completion of the study; or used medications known to interfere with creatinine elimination or tubular secretion.

Subjects with renal impairment were also excluded if they had systolic blood pressure (BP) $>180$ or $<90 \mathrm{mmHg}$ at screening; diastolic $\mathrm{BP}>110$ or $<50 \mathrm{mmHg}$ at screening; were currently receiving hemodialysis or peritoneal dialysis; or had abnormal electrocardiogram (ECG) results [sinus bradycardia $(<40 \mathrm{bpm})$ ] or a prolonged QT interval [Fridericia's correction formula $(\mathrm{QTcF}) \geq 500 \mathrm{~ms})]$ at screening or before dosing on day 1 . Subjects with normal renal function were also excluded if they had a systolic BP $>140$ or $<90 \mathrm{mmHg}$ at screening; diastolic $\mathrm{BP}>90$ or $<40 \mathrm{mmHg}$ at screening; abnormal ECG results [sinus bradycardia $(<$ $40 \mathrm{bpm}$ ) or prolonged QT interval (QTcF males $\geq 450 \mathrm{~ms}$, females $\geq 460 \mathrm{~ms}$ )] at screening or before dosing on day 1 ; or $\mathrm{CrCl}<90 \mathrm{~mL} / \mathrm{min}$.

\subsection{Pharmacokinetic Analysis}

Study endpoint plasma PK parameters included maximum plasma concentration $\left(C_{\max }\right)$, area under the plasma concentration-time curve (AUC) from time 0 to the last measurable observation $\left(\mathrm{AUC}_{0-T}\right), T_{\max }$, AUC to infinity (AUC inf), elimination half-life $\left(T_{1 / 2}\right)$, eGFR, $\mathrm{CrCl}$, and apparent oral clearance $(\mathrm{CL} / \mathrm{F})$. Pharmacokinetic parameters for urine included renal clearance (CLr), cumulative amantadine recovery in urine $\left(\mathrm{Ae}_{(0-\mathrm{t})}\right)$, and fraction of dose recovered in urine $\left(\mathrm{fe}_{(0-96)}\right)$.

Assays for amantadine were performed at Lambda Therapeutic Research, Inc. (Scarborough, ON, Canada) using high-performance liquid chromatography with tandem mass spectrometry detection with deuterium-labeled amantadine (amantadine-d6) as the internal standard.

The lower and upper limits of the assay were 2.076 and $1498.833 \mathrm{ng} / \mathrm{mL}$ for plasma and 19.854 and $2999.613 \mathrm{ng} /$ $\mathrm{mL}$ for urine, respectively. Concentration values below the limit of detection were reported as 0 .

\subsection{Planned Sample Size and Statistical Analysis}

This was an exploratory PK study with sufficient enrollment to allow the detection of PK differences across different levels of renal functioning. This study was not powered for inferential statistical analysis. It was determined that an 8 -subject control group and 8 subjects each in both test groups for renal impairment (moderate or severe) was sufficient to detect large enough PK differences to warrant dosage adjustment.

All PK parameters were calculated using the PK analysis set, which included all subjects who received the study drug. All statistical inferences were performed using the natural logarithmic transformation of $C_{\max }, \mathrm{AUC}_{0-T}$ and $\mathrm{AUC}_{\text {inf }}$, the observed $T_{1 / 2}, \mathrm{CLr}, \mathrm{Ae}_{(0-t)}$, and the rank transformation of $T_{\max }$. Statistical analyses were performed using both eGFR and Cockcroft-Gault estimates of $\mathrm{CrCl}$, with eGFR being used as the primary metric. All PK parameters were analyzed by regression analysis and, if found to be significant, were subject to analysis of variance (ANOVA).

\subsection{Pharmacokinetic Modeling}

A two-compartment PK population model was developed using NONMEM 7 with PDx-Pop5.2. It contained a depot and a central compartment with first-order and zero-order (duration) input processes followed by first-order elimination. This base model was enhanced with a covariate model that incorporated the effect of $\mathrm{CrCl}$ on the elimination rate constant $(k)$. Goodness-of-fit was evaluated by diagnostic plots.

Amantadine plasma concentration-time profiles were simulated using various administration intervals $(24,48$, $72,96,120$, and $168 \mathrm{~h}$ ) out to $960 \mathrm{~h}(1008 \mathrm{~h}$ for the weekly administration) with various $\mathrm{CrCl}$ values and dose regimens. Simulations were performed with the estimated pharmacokinetic model parameters.

Target amantadine plasma exposure was established by simulating a daily dose regimen of a $129-\mathrm{mg}$ amantadine ER tablet to a subject with normal $\mathrm{CrCl}$ values. Simulations of various dose regimens were carried out for subjects with varying levels of renal impairment. Recommended dose regimens were considered those that produced a plasma exposure profile similar to the target amantadine plasma profile.

\section{Results}

\subsection{Subject Baseline Characteristics}

The PK and safety population included 24 subjects $(8$ with normal renal function, 8 with moderate renal impairment, and 8 with severe renal impairment). All subjects completed the study, and none discontinued due to AEs. Demographic characteristics were similar across all three 
study groups (Table 1). Mean $\mathrm{CrCl}(\mathrm{mL} / \mathrm{min})$ and eGFR $\left(\mathrm{mL} / \mathrm{min} / 1.73 \mathrm{~m}^{2}\right)$, respectively, were 114.25 and 89.25 for subjects with normal renal function, 56.00 and 40.63 for subjects with moderate renal impairment, and 32.25 and 21.13 for subjects with severe renal impairment.

\subsection{Pharmacokinetic Results}

Plasma amantadine concentrations slowly increased over time following administration of the study drug (median $T_{\max }$ was $11.5 \mathrm{~h}$ for moderate renal impairment and $11 \mathrm{~h}$ for normal renal function and severe renal impairment) (Fig. 1). Predose amantadine plasma and urine concentrations were below the limit of detection in all samples. Table 2 shows the mean and coefficient of variation (CV) for relevant plasma $\mathrm{PK}$ parameters $\left(C_{\max }, \mathrm{AUC}_{0-T}\right.$ and $\left.\mathrm{AUC}_{\mathrm{inf}}, T_{\max }, T_{1 / 2}, \mathrm{CL} / \mathrm{F}, \mathrm{CLr}\right)$. All study groups had similar median $T_{\max }$ values. Renal impairment was associated with higher $C_{\max }$ and AUC values and lower CL/F and CLr values compared with their values in patients with normal renal function. When using either eGFR or $\mathrm{CrCl}$ as the main variable, regression analysis indicates that as renal impairment severity increases, $C_{\max }$ and AUC increase and CLr, $\mathrm{Ae}_{(0-t)}$, and CL/F decrease (Table 3). Regression analyses investigating the relationship between renal amantadine ER clearance and eGFR are shown in Fig. S2 of the ESM.

The amantadine renal clearance rate was $2618 \mathrm{~mL} / \mathrm{h}$ for subjects with severe impairment and $10,965 \mathrm{~mL} / \mathrm{h}$ in subjects with normal renal function. Within the first 4 days postdose, the fraction of amantadine dose excreted $\left(\mathrm{fe}_{(0-96)}\right)$ was $33 \%$ in subjects with severe renal impairment compared with $60.9 \%$ in subjects with normal renal function.
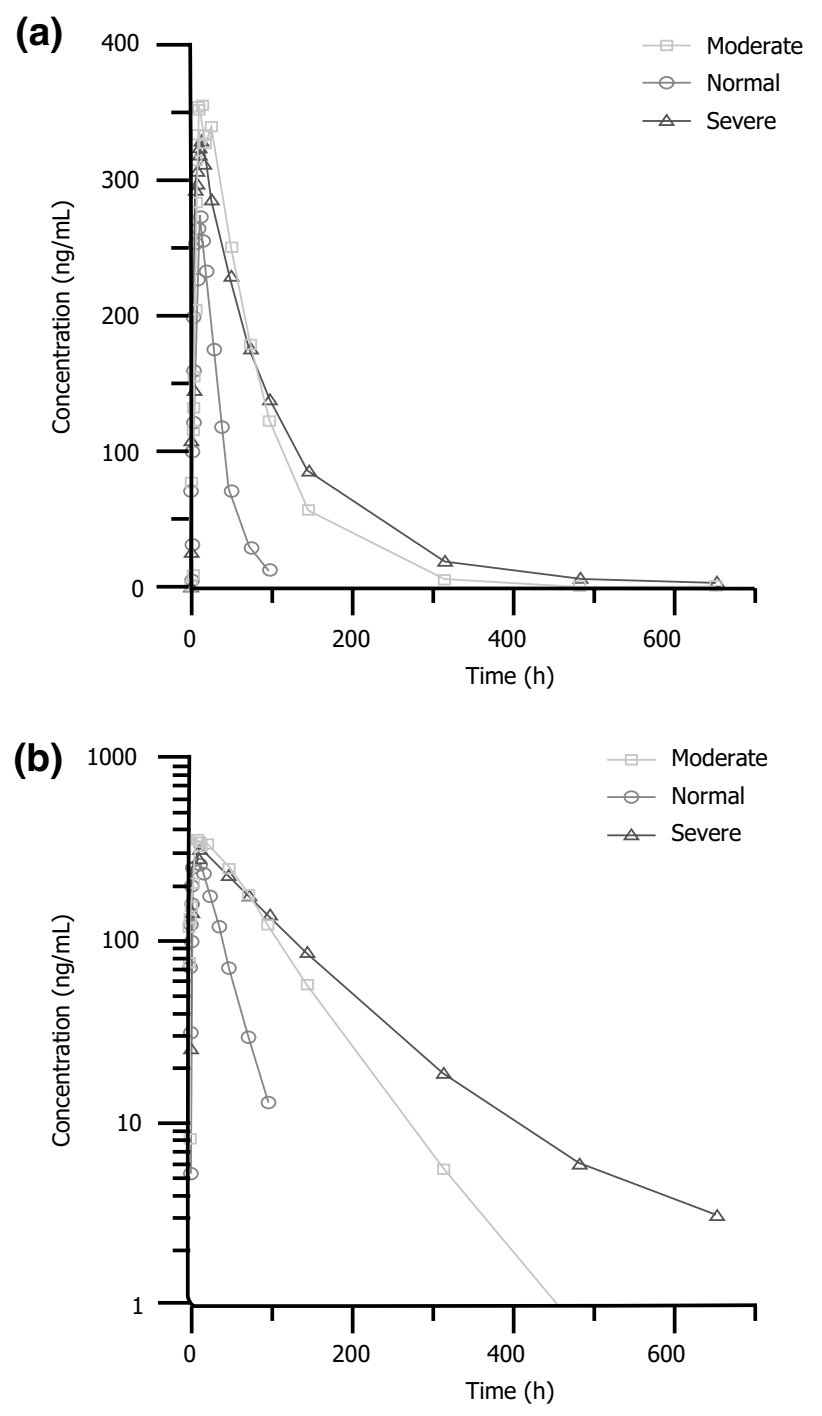

Fig. 1 Mean plasma amantadine concentration-time profile following a single oral dose of a 129-mg amantadine extended-release tablet in subjects with moderate and severe renal impairment and normal renal function: a linear scale; b semilogarithmic scale

Table 1 Summary of demographics

\begin{tabular}{lllll}
\hline Parameter & $\begin{array}{l}\text { Severe renal impairment } \\
(n=8)\end{array}$ & $\begin{array}{l}\text { Moderate renal impairment } \\
(n=8)\end{array}$ & Healthy $(n=8)$ & Overall $(n=24)$ \\
\hline $\begin{array}{l}\text { Age (years) } \\
\text { Sex }\end{array}$ & $57(11)$ & $63(12)$ & $51(7)$ & $57(11)$ \\
$\quad$ Male & & & $6(75.0)$ & $18(75.0)$ \\
Female & $6(75.0)$ & $6(75.0)$ & $2(25.0)$ & $6(25.0)$ \\
BMI $\left(\mathrm{kg} / \mathrm{m}^{2}\right)$ & $2(25.0)$ & $2(25.0)$ & $29.3(4.0)$ & $30.5(5.1)$ \\
$\mathrm{CrCl}(\mathrm{mL} / \mathrm{min})$ & $30.6(6.4)$ & $31.6(4.9)$ & $114.3(26.1)$ & - \\
eGFR $\left(\mathrm{mL} / \mathrm{min} / 1.73 \mathrm{~m}^{2}\right)$ & $32.3(17.7)$ & $56.0(20.2)$ & $89.3(10.6)$ & - \\
\hline
\end{tabular}

Data presented as mean (SD)

$B M I$ body mass index, $\mathrm{CrCl}$ creatinine clearance, $e G F R$ estimated glomerular filtration rate, $S D$ standard deviation

${ }^{\mathrm{a}} n(\%)$ 
Table 2 Summary of pharmacokinetic parameters of amantadine following a single oral dose of a 129-mg amantadine extended-release tablet in subjects with moderate or severe renal impairment and normal renal function

\begin{tabular}{lcrr}
\hline Parameter (units) & $\begin{array}{l}\text { Severe renal impairment }(n \\
=8)\end{array}$ & $\begin{array}{l}\text { Moderate renal impairment } \\
(n=8)\end{array}$ & Healthy $(n=8)$ \\
\hline$C_{\max }(\mathrm{ng} / \mathrm{mL})$ & $344.94(22.8 \%)$ & $380.01(21.4 \%)$ & $281.29(26.4 \%)$ \\
$T_{\max }(\mathrm{h})^{\mathrm{a}}$ & $11.00(6.0-14.0)$ & $11.50(9.0-24.0)$ & $11.00(8.0-12.2)$ \\
$\mathrm{AUC}_{\text {inf }}(\mathrm{ng} \cdot \mathrm{h} / \mathrm{mL})$ & $39,031.65(39.9 \%)$ & $33,066.14(31.6 \%)$ & $10,108.66(26.9 \%)$ \\
Oral clearance $(\mathrm{L} / \mathrm{h})$ & $4.56(29.9 \%)$ & $5.48(45.2 \%)$ & $17.37(40.3 \%)$ \\
Elimination half-life $(\mathrm{h})$ & $116.27(46.6 \%)$ & $47.19(31.4 \%)$ & $18.47(13.2 \%)$ \\
Cumulative amantadine recovered in urine $(\mathrm{mg})$ & $52.93(24.8 \%)$ & $32.57(20.5 \%)$ & $97.46(3.7 \%)$ \\
Renal clearance $(\mathrm{mL} / \mathrm{h})$ & $2618.22(34.5 \%)$ & $459.27(47.0 \%)$ & $10,965.10(41.3 \%)$ \\
Fraction of dose recovered in urine $(\%)$ & $33.08(24.8 \%)$ & $65.35(20.5 \%)$ & $60.91(3.7 \%)$ \\
\hline
\end{tabular}

Data presented as mean (coefficient of variation)

$A U C_{\text {inf }}$ area under the concentration-time curve (AUC) from time 0 to infinity, $C_{\max }$ maximum plasma concentration, $T_{\max }$ time to maximum concentration

${ }^{a}$ Median (range)

Table 3 Summary of statistical analysis of amantadine pharmacokinetic parameters following a single oral dose of a 129-mg amantadine extended-release tablet in subjects with moderate and severe renal impairment and normal renal function

\begin{tabular}{llclccc}
\hline Parameter & Group & Geometric LS mean & Comparison & Adjusted $p$ value & Ratio & 90\% CI (min, max) \\
\hline$C_{\text {max }}$ & Severe & 336.772 & Severe vs moderate & 0.6250 & 90.42 & $(70.75,115.56)$ \\
& Moderate & 372.449 & Severe vs normal & 0.2441 & 123.51 & $(93.71,162.80)$ \\
& Healthy & 272.664 & Moderate vs normal & 0.0468 & 136.60 & $(104.78,178.07)$ \\
AUC & Severe & $36,796.005$ & Severe vs moderate & 0.6544 & 117.49 & $(79.19,174.33)$ \\
& Moderate & $31,317.010$ & Severe vs normal & $<0.0001$ & 378.90 & $(262.52,546.87)$ \\
& Healthy & 9711.364 & Moderate vs normal & $<0.0001$ & 322.48 & $(220.42,471.80)$ \\
Cumulative amantadine & Severe & 51.403 & Severe vs moderate & 0.0270 & 72.08 & $(55.98,92.79)$ \\
recovered in urine & Moderate & 71.319 & Severe vs normal & $<0.0001$ & 52.77 & $(43.01,64.76)$ \\
& Healthy & 97.402 & Moderate vs normal & 0.0007 & 73.22 & $(62.80,85.38)$ \\
Renal clearance & Severe & 2445.165 & Severe vs moderate & 0.4282 & 76.20 & $(47.86,121.33)$ \\
& Moderate & 3208.907 & Severe vs normal & $<0.0001$ & 23.56 & $(15.61,35.55)$ \\
& Healthy & $10,379.461$ & Moderate vs normal & $<0.0001$ & 30.92 & $(20.36,46.95)$ \\
\hline
\end{tabular}

$A U C_{\text {inf }}$ area under the plasma concentration-time curve to infinity, $C I$ confidence interval, $C_{\max }$ maximum plasma concentration, $L S$ least squares, max maximum, min minimum

\subsection{Pharmacokinetic Modeling/simulation}

Parameters for simulated steady-state PK models are shown in Table 1 of the ESM. The identified target exposure ranges as determined from subjects with normal renal function for $C_{\text {max }}$, plasma concentration at the end of a dose interval $\left(C_{\min }\right)$, and average steady-state plasma concentration during a dose interval $\left(C_{\text {avg }}\right)$ were $385-610 \mathrm{ng} / \mathrm{mL}, 183-401 \mathrm{ng} /$ $\mathrm{mL}$, and $300-526 \mathrm{ng} / \mathrm{mL}$, respectively. Final dosage recommendations based on the modeling of subjects with moderate to severe renal impairment are outlined in Table 4. It is recommended that for subjects with moderate to severe renal impairment, amantadine ER $129 \mathrm{mg}$ should be taken every 48-168 h, depending on the degree of renal impairment.

\section{Discussion}

Subjects with moderate or severe renal impairment had significantly increased plasma levels of amantadine following administration of a single dose of $129 \mathrm{mg}$ amantadine ER. In this study, all study groups had similar median $T_{\max }$ values, indicating that renal impairment did not influence amantadine absorption. However, reduced clearance rates in subjects with moderate to severe renal impairment resulted in a three- to fourfold increase in overall amantadine exposure. Reductions in amantadine clearance directly corresponded to the degree of renal impairment. The PK modeling undertaken in this work provides temporal variation guidelines 
Table 4 Amantadine extended-release tablet dose regimens recommended for subjects with impaired renal function

\begin{tabular}{llll}
\hline Renal function group & $\mathrm{CrCl}(\mathrm{mL} / \mathrm{min})$ & Dose $(\mathrm{mg})$ & $\begin{array}{l}\text { Dose admin- } \\
\text { istration } \\
\text { frequency }\end{array}$ \\
\hline Normal & $\geq 90$ & 129 & Every $24 \mathrm{~h}$ \\
Mild impairment & $60-89$ & 129 & Every $48 \mathrm{~h}$ \\
Moderate impairment & $45-59$ & 129 & Every $72 \mathrm{~h}$ \\
& $30-44$ & 129 & Every $96 \mathrm{~h}$ \\
Severe impairment & $22-29$ & 129 & Every $120 \mathrm{~h}$ \\
(not on dialysis) & $15-21$ & 129 & Every $168 \mathrm{~h}$ \\
\hline
\end{tabular}

$\mathrm{CrCl}$ creatinine clearance

when dosing renally impaired subjects such that subject exposure remains consistent with that of a subject with normal renal function taking once-daily amantadine ER.

Amantadine ER is approved for the treatment of PD and drug-induced extrapyramidal reactions in adults. In subjects with normal renal function, the steady-state amantadine IR plasma concentration peaks at $0.63 \mu \mathrm{g} / \mathrm{mL}$ after $2.1 \mathrm{~h}$ following 5 days of a twice-daily oral $81-\mathrm{mg}$ dose $(100 \mathrm{mg}$ amantadine $\mathrm{HCl}$ ) [7]. Here, we report that subjects with normal renal function receiving a single $129-\mathrm{mg}$ dose of the ER formulation reached a peak plasma concentration of $0.28(\mathrm{CV} 26.4 \%) \mu \mathrm{g} / \mathrm{mL}$ at a median time of 11.0 (range 8.0-12.2) h postdose.

Amantadine plasma concentration has been correlated with clinical efficacy in nonhuman primates [12]. However, increased amantadine exposure can also lead to increases in AEs. The incidence of amantadine toxicity in subjects with renal impairment is well documented and includes symptoms such as ataxia, dizziness, depression, aggression, confusion, slurred speech, convulsions, or blurred vision [10, 13-16]. Amantadine IR clearance is known to be slower in individuals with renal impairment, with a $\mathrm{CrCl}<40 \mathrm{~mL} /$ $\mathrm{min} / 1.73 \mathrm{~m}^{2}$ resulting in an at least two- to threefold increase in plasma half-life [2]. This study shows that subjects with moderate renal impairment $\left(30-59 \mathrm{~mL} / \mathrm{min} / 1.73 \mathrm{~m}^{2}\right)$ receiving a single 129-mg oral dose of amantadine ER have a roughly 2.5 -fold increase in $T_{1 / 2}$ relative to subjects with normal renal function.

This study is limited by the exclusion of subjects on hemodialysis. Previous work has shown that amantadine removal by hemodialysis is negligible, with a 4-h hemodialysis session removing less than $5 \%$ of the amantadine [10, 17]. Subjects with renal impairment who are taking amantadine ER should be closely monitored for AEs.

\section{Conclusions}

Overall, results from this single-dose study indicate that subjects with impaired renal function exhibit significant deficits in amantadine clearance. If a typical dosing regimen is followed, it could lead to an accumulation of amantadine in the bloodstream, thus increasing the risk of AEs and amantadine toxicity. It is recommended that subjects with normal renal function initiate treatment by taking $129 \mathrm{mg}$ amantadine ER once daily in the morning and then increase dosing in weekly intervals as needed [6]. Amantadine ER is contraindicated for subjects with $\mathrm{CrCl}<15 \mathrm{~mL} / \mathrm{min}$ [6]. The results of this study indicate that subjects with renal impairment should initiate and maintain treatment with $129 \mathrm{mg}$ taken at the dose frequency that corresponds to their degree of renal impairment. Subjects with mild renal impairment $(\mathrm{CrCl}$ 60-89 $\mathrm{mL} / \mathrm{min}$ ) should take $129 \mathrm{mg}$ amantadine ER every $48 \mathrm{~h}$, subjects with moderate impairment should take it every $72(\mathrm{CrCl} 45-59 \mathrm{~mL} / \mathrm{min})$ to $96(\mathrm{CrCl} \mathrm{30-44} \mathrm{mL} / \mathrm{min}) \mathrm{h}$, and subjects with severe renal impairment should take it every $120(\mathrm{CrCl} 22-29 \mathrm{~mL} / \mathrm{min})$ to $168(\mathrm{CrCl} 15-21 \mathrm{~mL} / \mathrm{min}) \mathrm{h}$.

Acknowledgements Medical writing and editorial support were provided by Alicia Salinero, PhD, of AlphaBioCom, LLC (King of Prussia, PA, USA) and funded by Osmotica Pharmaceutical US LLC (Bridgewater, NJ, USA). We thank Eric Sicard, MD and Benoît Deschamps, MD (Algorithme Pharma Inc., Laval, Quebec, Canada) for clinical study and pharmacokinetic analysis, and Mark Warren (Lambda Therapeutic Research Inc., Toronto, Ontario, Canada) for bioanalytic analysis.

Author Contributions AD is VP of Program Management, TD is EVP of R\&D, and DJ is VP of Clinical Operations and Medical Affairs. VP was a principal investigator in this study. TD and CD were responsible for statistical analysis. All authors contributed equally to the drafting and editing of the paper, and all authors approved the final content.

\section{Compliance with Ethical Standards}

Funding This study was funded by Osmotica Pharmaceutical US LLC. Open access fees were provided by Osmotica Pharmaceutical US LLC. Medical writing and editorial support were provided by Alicia Salinero, $\mathrm{PhD}$, of AlphaBioCom, LLC (King of Prussia, PA, USA) and funded by Osmotica Pharmaceutical US LLC (Bridgewater, NJ, USA).

Conflict of interest Tina deVries, Angela Dentiste, and David Jacobs are employees of Osmotica Pharmaceutical US LLC and have equity interests in Osmotica Pharmaceuticals plc, the ultimate parent company of Osmotica Pharmaceutical US LLC. Clifford Di Lea is an employee of Aclairo Pharmaceutical Development Group, Inc., and received study funding from Osmotica Pharmaceutical US LLC. Vincent Pichette received funding from Algorithme Pharma to conduct part of this study. 
Ethical approval This study was approved by institutional review boards and conducted in compliance with Good Clinical Practice regulations and guidelines as defined by the International Conference of Harmonization. This work complies with ethical principles outlined in the US Code of Federal Regulations, Title 21, Part 50 (21CFR50), and European Union Directive 2001/20/EC. A letter of nonobjection (control number 184363) was obtained from Canadian authorities.

Informed consent All subjects signed informed consent forms after voluntarily agreeing to participate in the study.

Full financial disclosures $\mathrm{TD}, \mathrm{AD}$, and $\mathrm{DJ}$ are funded by Osmotica Pharmaceutical US LLC. CD is funded by Aclairo Pharmaceutical Development Group, Inc., and Osmotica Pharmaceutical US LLC. VP received a grant from Algorithme Pharma to conduct part of this study.

Open Access This article is distributed under the terms of the Creative Commons Attribution-NonCommercial 4.0 International License (http://creativecommons.org/licenses/by-nc/4.0/), which permits any noncommercial use, distribution, and reproduction in any medium, provided you give appropriate credit to the original author(s) and the source, provide a link to the Creative Commons license, and indicate if changes were made.

\section{References}

1. Endo Pharmaceuticals. Prescribing information for SYMMETREL $^{\circledR}$ (amantadine hydrochloride, USP) tablets and syrup. 2009. https://www.accessdata.fda.gov/drugsatfda_docs/ label/2009/016023s041,018101s016lbl.pdf. Accessed 7 Nov 2017.

2. Aoki FY, Sitar DS. Clinical pharmacokinetics of amantadine hydrochloride. Clin Pharmacokinet. 1988;14(1):35-51.

3. Muller T, Kuhn W, Quack G, Przuntek H. Intravenous application of amantadine and antiparkinsonian efficacy in Parkinsonian patients. J Neural Transm Suppl. 1995;46:407-13.

4. Somani SK, Degelau J, Cooper SL, Guay DR, Ehresman D, Zaske D. Comparison of pharmacokinetic and safety profiles of amantadine 50- and 100-mg daily doses in elderly nursing home residents. Pharmacotherapy. 1991;11(6):460-6.
5. Vargus-Adams JN, McMahon MA, Michaud LJ, Bean J, Vinks AA. Pharmacokinetics of amantadine in children with impaired consciousness due to acquired brain injury: preliminary findings using a sparse-sampling technique. PM R. 2010;2(1):37-42.

6. Osmotica Pharmaceutical. Prescribing information for OSMOLEX ER ${ }^{\mathrm{TM}}$ (amantadine): extended-release tablets, for oral use. 2018. https://www.accessdata.fda.gov/drugsatfda_docs/label /2018/209410s000lbl.pdf. Accessed 10 Mar 2018.

7. Morrison D, Roy S, Rayner C, Amer A, Howard D, Smith JR, et al. A randomized, crossover study to evaluate the pharmacokinetics of amantadine and oseltamivir administered alone and in combination. PLoS One. 2007;2(12):e1305.

8. Liu P, Cheng PJ, Ing TS, Daugirdas JT, Jeevanandhan R, Soung LS, et al. In vitro binding of amantadine to plasma proteins. Clin Neuropharmacol. 1984;7(2):149-51.

9. Deleu D, Northway MG, Hanssens Y. Clinical pharmacokinetic and pharmacodynamic properties of drugs used in the treatment of Parkinson's disease. Clin Pharmacokinet. 2002;41(4):261-309.

10. Horadam VW, Sharp JG, Smilack JD, McAnalley BH, Garriott JC, Stephens MK, et al. Pharmacokinetics of amantadine hydrochloride in subjects with normal and impaired renal function. Ann Intern Med. 1981;94(4 pt 1):454-8.

11. Cockcroft DW, Gault MH. Prediction of creatinine clearance from serum creatinine. Nephron. 1976;16(1):31-41.

12. Elkurd MT, Bahroo LB, Pahwa R. The role of extended-release amantadine for the treatment of dyskinesia in Parkinson's disease patients. Neurodegener Dis Manag. 2018;8(2):73-80.

13. Miller KS, Miller JM. Toxic effects of amantadine in patients with renal failure. Chest. 1994;105(5):1630.

14. Nakai K, Takeda K, Kimura H, Miura S, Maeda A. Obstructive acute renal failure related to amantadine intoxication. Am J Emerg Med. 2009;27(3):371.e5-7.

15. Nakata M, Ito S, Shirai W, Hattori T. Severe reversible neurological complications following amantadine treatment in three elderly patients with renal insufficiency. Eur Neurol. 2006;56(1):59-61.

16. Wu MJ, Ing TS, Soung LS, Daugirdas JT, Hano JE, Gandhi VC. Amantadine hydrochloride pharmacokinetics in patients with impaired renal function. Clin Nephrol. 1982;17(1):19-23.

17. Soung LS, Ing TS, Daugirdas JT, Wu MJ, Gandhi VC, Ivanovich PT, et al. Amantadine hydrochloride pharmacokinetics in hemodialysis patients. Ann Intern Med. 1980;93(1):46-9. 